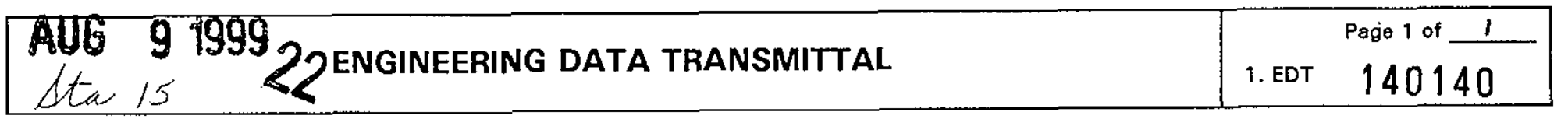

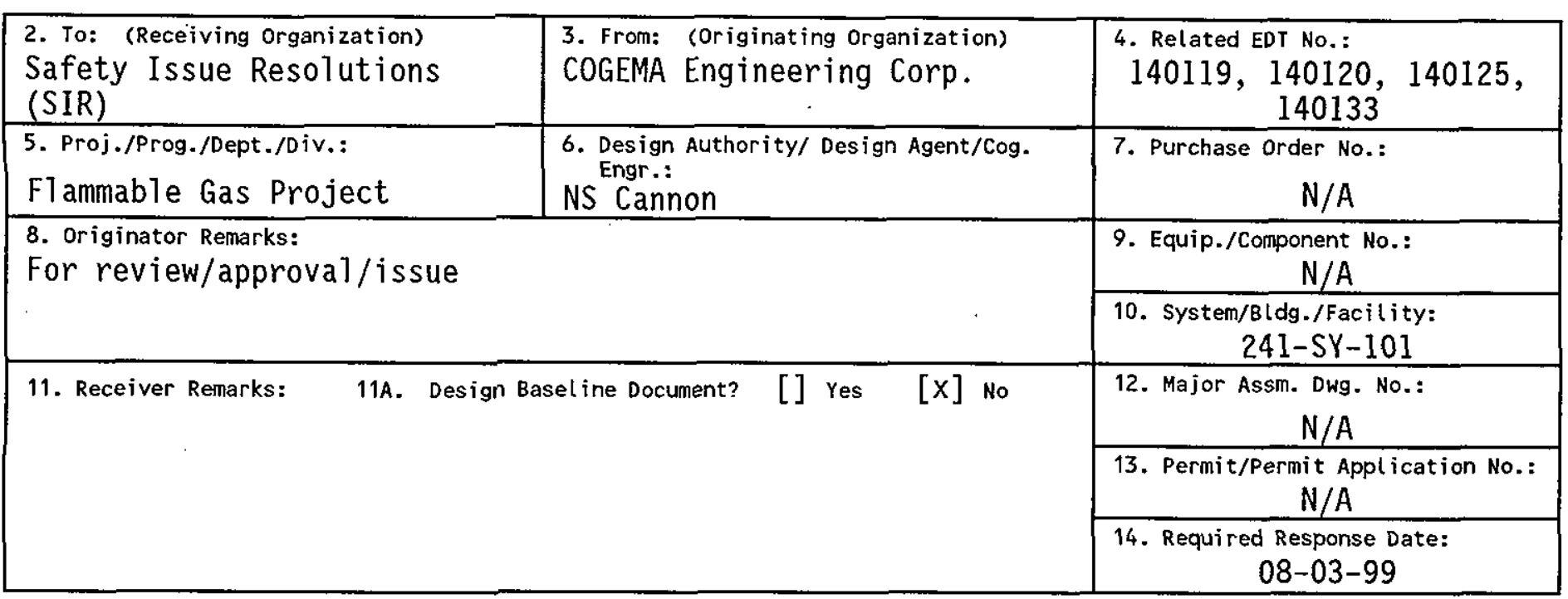

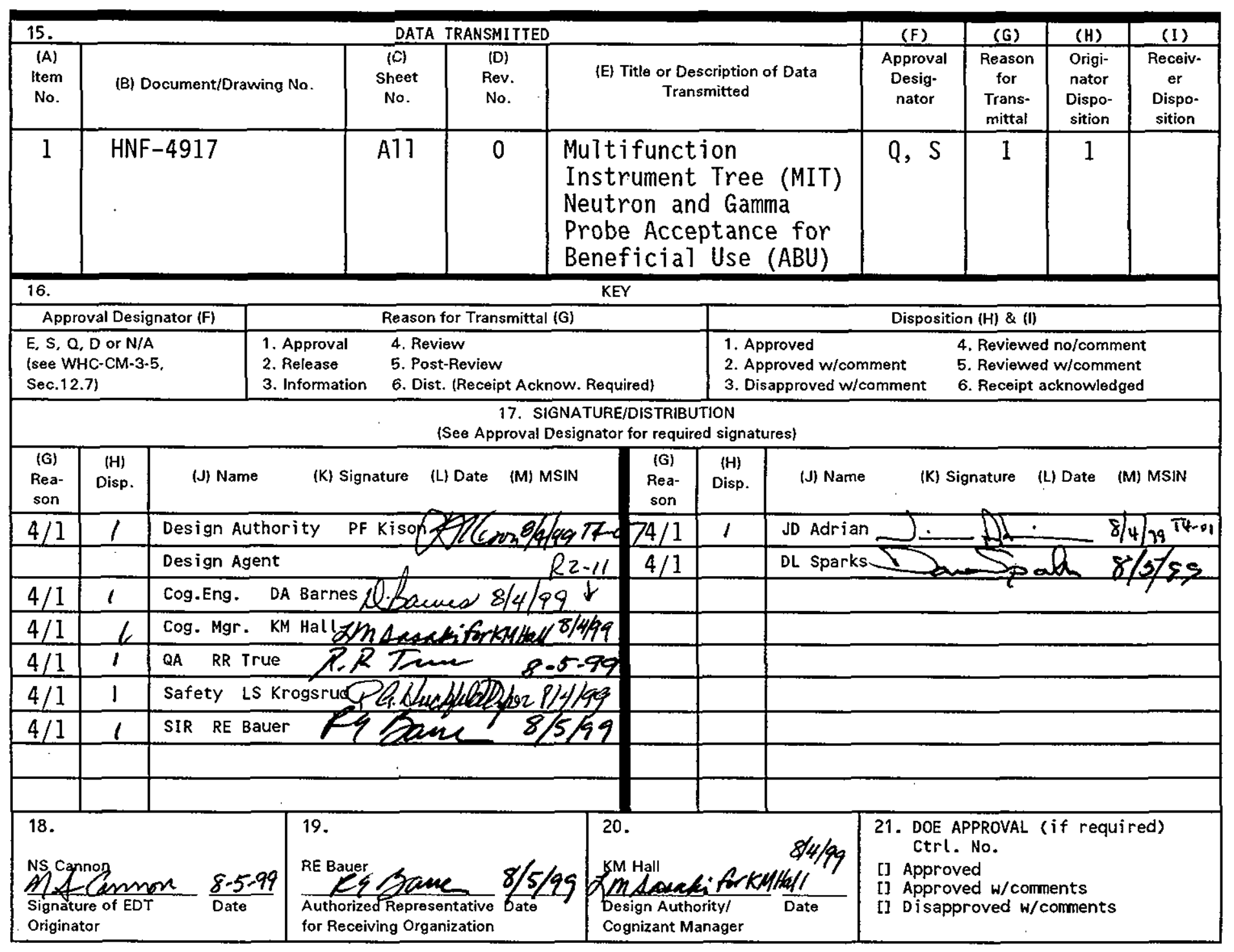

BD-7400-172-2 (05/96) GEF097 


\section{Multifunction Instrument Tree (MIT) Neutron and Gamma Probe Acceptance for Beneficail Use (ABU)}

N. S. Cannon

COGEMA Engineering Corporation, Richland, WA 99352

U.S. Department of Energy Contract DE-AC06-96RL13200

$\begin{array}{lll}\text { EDT/ECN: } & 140140 & \text { UC: } 2070 \\ \text { Org Code: } & 08 E 00 & \text { Charge Code: } 106033 \text { BA10 - HN920201 } \\ \text { B\&R Code: } & \text { EW3120074 } & \text { Total Pages: } 12\end{array}$

Key Words: acceptance test, operational test, ATP/OTP, OTR, 241-SY-101, interstitial liquid level (ILL), neutron, gamma, probe, multifunction instrument tree (MIT), flammable gas, inventory, hazard, liquid observation well (LOW)

Abstract: This report documents the completion of the final Acceptance for Beneficial Use (ABU) of the MIT neutron and gamma probes for application at Tank 241-SY-101.

TRADEMARK DISCLAIMER. Reference herein to any specific commercial product, process, or service by trade name, tradenark, manufacturer, or otherwise, does not necessarily constitute or imply its endorsement, recommendation, or favoring by the United States Government or any agency thereof or its contractors or subcontractors.

Printed in the United States of America. To obtain copies of this document, contact: Document Control Services, P.O. Box 950, Mailstop H6-08, Richland WA 99352, Phone (509) 372-2420; Fax (509) 376-4989.
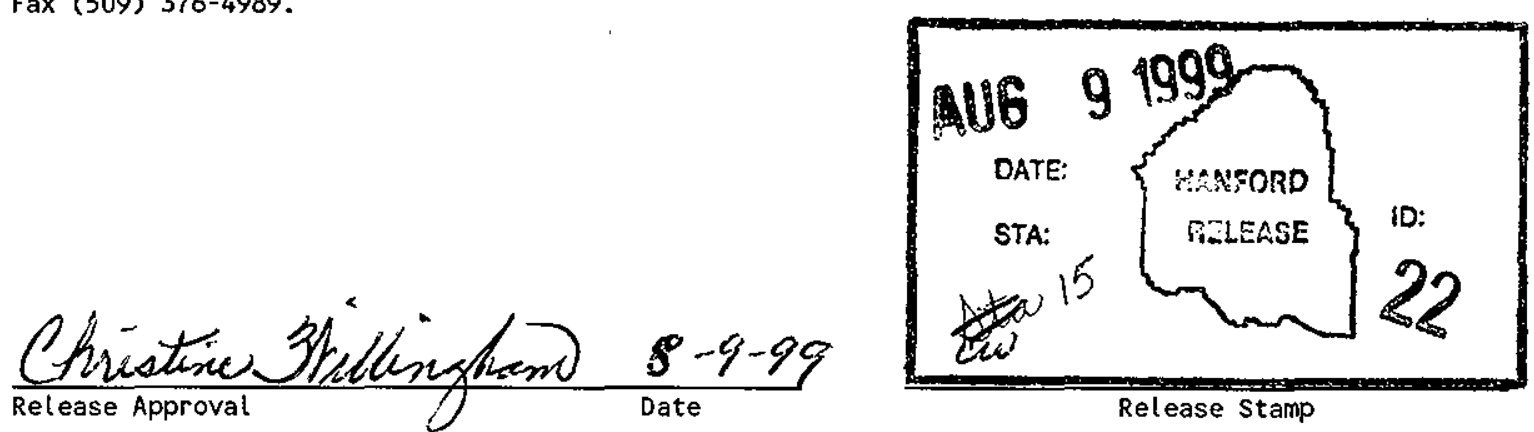


\section{MULTIFUNCTION INSTRUMENT TREE (MIT) NEUTRON AND GAMMA PROBE ACCEPTANCE FOR BENEFICIAL USE (ABU)}

N. Scott Cannon

COGEMA Engineering Corporation

August 1999 
HNF-4917, Rev. 0

\section{TABLE OF CONTENTS}

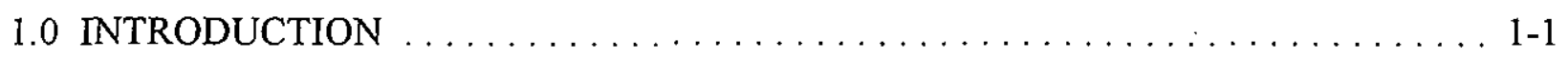

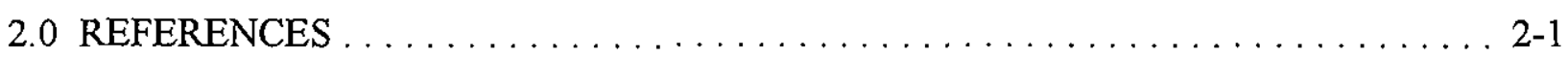

3.0 MIT PROBE ACCEPTANCE FOR BENEFICIAL USE (FINAL) . . . . . . . . . 3-1 APPENDIX

Planned ABU from Engineering Task Plan (HNF-3322) . . . . . . . . . . A-1 
HNF-4917, Rev. 0

\section{MULTIFUNCTION INSTRUMENT TREE (MIT) NEUTRON AND GAMMA PROBE ACCEPTANCE FOR BENEFICIAL USE (ABU)}

\subsection{INTRODUCTION}

The multifunction instrument tree (MIT) probe program has been developed to modify existing Liquid Observation Well (LOW) neutron and gamma probes for use in the validation shafts of the two MTS installed in Tank 241-SY-101. One of the program objectives is that the modified MIT probes be completely compatible with the existing LOW van instrumentation and procedures. The major program objective is to produce neutron and gamma scans from Tank 241-SY-101 that would assist in evaluating waste feature structure and elevation. The MIT probe program is described in greater detail in the engineering task plan (HNF-3322).

In accordance with the engineering task plan, a test plan (HNF-3595) was written, reduced diameter (allowing insertion into the MIT validation tube) neutron and gamma probes were acquired, an acceptance and operational test procedure (HNF-3838) was written, acceptance and operational testing of the MIT probes was performed, and a report of these test results (HNF-4369) has been issued.

A number of neutron and gamma probe scans have been obtained from the Tank 241-SY-101 MITs, starting on February 8, 1999, in cooperation with Operations. Now that the MIT probes are fully demonstrated, this document transfers ownership of these probes to Operations, utilizing the final acceptance for beneficial use (ABU) form that follows in Section 3.0. For comparison, the original $\mathrm{ABU}$ planned in the engineering task plan is provided in the Appendix. 
HNF-4917, Rev. 0

\subsection{REFERENCES}

Cannon, N. S., 1998a, Engineering Task Plan for Determining the Interstitial Liquid Level in Tank 241-SY-101 Utilizing a Neutron Probe in the Multifunction Instrument Tree, HNF-3322, Rev. 0, COGEMA Engineering Corp. For Fluor Daniel Hanford Inc., Richland, Washington.

Cannon, N. S., 1998b, Test Plan for Neutron Probe Application to Tank 241-SY-101 MITs, HNF-3595, Rev. 0, COGEMA Engineering Corp. for Fluor Daniel Hanford, Inc., Richland, Washington.

Cannon, N. S., 1999a, Acceptance and Operational Test Procedure for Neutron and Gamma Probe Application to Tank 241-SY-101 MITs, HNF-3838, Rev. 1, COGEMA Engineering Corp. for Flour Daniel Hanford Inc., Richland, Washington.

Cannon, N. S., 1999b, Acceptance and Operational Test Report for Neutron and Gamma Probe Application to Tank 241-SY-10I MITs, HNF-4369, Rev. 0, COGEMA Engineering Corp. for Flour Daniel Hanford Inc., Richland, Washington

LMHC, 1998, Liquid Observation Well (LOW) Surveillance Van Startup and Operation Procedure, TO-040-333, Rev. A-4 (or latest revision), Lockheed Martin Hanford Corporation for Fluor Daniel Hanford, Inc., Richland, Washington.

LOW Surveillance Van Maintenance Manual, Rev. A-3, 1998, Greenspan, Inc., Houston, Texas. 


\subsection{MIT PROBE ACCEPTANCE FOR BENEFICIAL USE (FINAL)}

\begin{tabular}{|c|c|c|c|c|c|}
\hline \multicolumn{6}{|c|}{ ACCEPTANCE FOR BENEFICIAL USE (ABU) } \\
\hline $\begin{array}{l}\text { 3. Document No. } \\
\text { HNF-4917 }\end{array}$ & $\begin{array}{l}\text { 4. Project No. } \\
\text { NA }\end{array}$ & $\begin{array}{l}\text { 5. Ssc } \\
\text { Designator } \\
\text { GS }\end{array}$ & $\begin{array}{l}\text { 6.System/BIdg/ } \\
\text { Facility } \\
241-S Y-101\end{array}$ & $\begin{array}{l}\text { 7.Equip/Component } \\
\text { No. }\end{array}$ & $\begin{array}{l}\text { 8. ECN/EDT No. } \\
\text { EDT } 140140\end{array}$ \\
\hline 9. Final ABU $(x)$ & \multicolumn{2}{|c|}{ Partial ABU ( ) } & \multicolumn{3}{|l|}{ 10. ATP Rerun Required } \\
\hline \multicolumn{6}{|c|}{$\begin{array}{l}\text { 11. Description of Work } \\
\text { Modify Neutron/Gamma Probes to operate with the existing LOW Surveillance Vans at Tank } 241-S Y-101 \text { MITs in } \\
\text { accordance with procedure TO-040-333. These modified probes will be referred to as the 'MIT probes'. }\end{array}$} \\
\hline \multicolumn{6}{|c|}{$\begin{array}{l}\text { This ABU documents the completion of all activities necessary for the deployment of MIT probes with } \\
\text { the existing LOW vans at Tank } 241-S Y-101 \mathrm{MITS} \text {. }\end{array}$} \\
\hline \multicolumn{6}{|c|}{$\begin{array}{l}\text { Completed items include the engineering task plan (HNF-3322), procurement of the MIT probes, the } \\
\text { acceptance/operational test procedure (HNF-3838, Rev. 1), and the acceptance/operational test report } \\
\text { (HNF-4369) }\end{array}$} \\
\hline 12. Description of & to be Completed & $\begin{array}{l}\text { 13. Scheduled } \\
\text { Completion Date }\end{array}$ & $\begin{array}{l}\text { 14. Responsibility for } \\
\text { Completion }\end{array}$ & 15. Impact on Operat & ns/Safety \\
\hline \multicolumn{2}{|c|}{ NA } & NA & NA & \multicolumn{2}{|c|}{ NA } \\
\hline
\end{tabular}

16. Documents or Other Items/Tasks to be Updated

17. Funding Source
18. Document Number 18. Document Number

\section{Responsibility for Completion}

20. Required prior to final $A B U$ (Yes/No)

\section{IRAINING:}

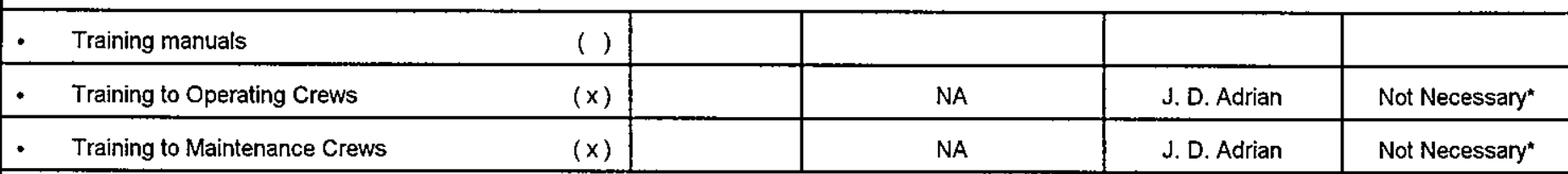

\section{ENGINEERING:}

\begin{tabular}{|lr|r|r|r|r|}
\hline - Engineering Task Plan & $(x)$ & & HNF-3322 & N. S. Cannon & Completed \\
\hline - Engineering Procedure & $($ ) & & & & \\
\hline Final Safety Analysis Report (FSAR) & $($ ) & & & & \\
\hline Safety Assessment (SA) & $($ ) & & & & \\
\hline
\end{tabular}


HNF-4917, Rev. 0

\begin{tabular}{|c|c|c|c|c|}
\hline 16. Documents or Other Items/Tasks to be Updated & \multirow{2}{*}{$\begin{array}{l}\text { 17. Funding } \\
\text { Source }\end{array}$} & \multirow[t]{2}{*}{ 18. Document Number } & \multirow{2}{*}{$\begin{array}{l}\text { 19. Responsibility } \\
\text { for Completion }\end{array}$} & \multirow{2}{*}{$\begin{array}{l}\text { 20. Required } \\
\text { prior to final ABU } \\
\text { (Yes/No) }\end{array}$} \\
\hline - Functional Design Criteria (FDC) ( ) & & & & \\
\hline - $\quad$ Design Review Report & & & & \\
\hline - $\quad$ Conceptual Design Report (CDR) & & & & \\
\hline $\begin{array}{l}\text { - Supplemental Design Requirements Document } \\
\text { (SDRD) }\end{array}$ & & . & & \\
\hline - System Design Description (SDD) ( ) & & & & \\
\hline $\begin{array}{l}\text { Test procedures/specifications } \\
\text { Test Report }\end{array}$ & & $\begin{array}{c}\text { ATP/OTP (HNF-3838 } \\
\text { Rev.1) } \\
\text { ATR/OTR (HNF-4369 } \\
\text { Rev. 0) }\end{array}$ & N. S. Cannon & Completed \\
\hline - Safety Equipment List & & & & \\
\hline - Environmental Impact Statement & & & & \\
\hline - Environmental Report & & & & \\
\hline - Environmental Permits & & & & \\
\hline - Stress/Seismic Analysis & & & & \\
\hline - Stress/Design Report & & & & \\
\hline - Equipment Specification & & & & \\
\hline - Procurement Specification & & $\begin{array}{l}\text { Included in PR A31978 \& } \\
\qquad \text { A31980 }\end{array}$ & N. S. Cannon & Completed \\
\hline - Construction Specification & & & & \\
\hline - Essential Material Specification & & & & \\
\hline - Technical Specification & & & & \\
\hline - As-built Drawing & & $\begin{array}{l}\mathrm{H}-2-829671 \text { and } E C N \\
650901 \text { for } \mathrm{H}-2-75401\end{array}$ & P. R. Deichelborer & Completed \\
\hline - Interface Control Drawing & & & & \\
\hline - Computer Software & & & & \\
\hline \multicolumn{5}{|l|}{ OPERATIONS: } \\
\hline - Operating and Maintenance Manuals & & & & \\
\hline - Operating Procedures & & TO-040-333* & D. A. Barnes & Completed \\
\hline - Operation Instructions & & & & \\
\hline - Calibration Procedures & & TO-040-333** & D. A. Barnes & Completed \\
\hline
\end{tabular}


HNF-4917, Rev. 0

\begin{tabular}{|c|c|c|c|c|}
\hline 16. Documents or Other Items/Tasks to be Updated & $\begin{array}{l}\text { 17. Funding } \\
\text { Source }\end{array}$ & 18. Document Number & $\begin{array}{l}\text { 19. Responsibility } \\
\text { for Completion }\end{array}$ & $\begin{array}{l}\text { 20. Required } \\
\text { prior to final ABU } \\
(\text { Yes } / \mathrm{No})\end{array}$ \\
\hline - Preventative Maintenance Procedures & & & . & \\
\hline - Operations Safety Requirements & & & & \\
\hline \multicolumn{5}{|l|}{ QUALITY ASSURANCE: } \\
\hline - Inspection Plan & & $\begin{array}{l}\text { Part of ATP/OTP } \\
\text { (HNF-3838, Rev. 1) }\end{array}$ & R. R. True & Completed \\
\hline - $\quad$ QAPP & & & & \\
\hline - $\quad$ QAP JP & & & & \\
\hline - NEC Inspection & & & & \\
\hline \multicolumn{5}{|l|}{ PROCUREMENT ACTIVITIES: } \\
\hline - Vendor Information & & $\begin{array}{l}\text { Vendor Maintenance } \\
\text { Manual, } 1998\end{array}$ & N. S. Cannon & $\begin{array}{l}\text { Supplied to End } \\
\text { User }\end{array}$ \\
\hline - $\quad$ Material List & & & & \\
\hline - $\quad$ Spare Parts & & & & \\
\hline - $\quad$ Purchase Requisition(s) & & $\begin{array}{l}\text { PR A31978 \& A319830 } \\
\text { (PO 1075 \& 1076) }\end{array}$ & N. S. Cannon & $\begin{array}{c}\text { Received \& } \\
\text { Inspected }\end{array}$ \\
\hline - $\quad$ ALARA Management Worksheet & & & & \\
\hline - Other Commercial Grade Item forms & & & & \\
\hline
\end{tabular}

* No changes were required for the actual operation or maintenance procedures of the probes, so that training wasn't necessary. This is primarily because the $1.5 \mathrm{Ci}$ neutron source was found adequate, so that use of the $3 \mathrm{Ci}$ source isn't required.

** No changes were required in the TO-040-333 procedure to operate the MIT probes. 
HNF-4917, Rev. 0

PROJECTS: CHARACTERIZATION OPERATIONS AND ENGINEERING

\section{Signature}

Date

Cog/Field Engineer

DA Barnes
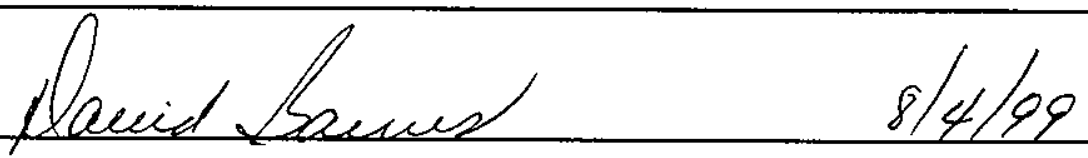

Cog/Field Engineer

Cog/Field Engineer

Cog/Field Mgr. KM Hall

finsackifor KM Hall

$8 / 4 / 99$

QA RR True

$\mathbb{R} R$ True

8.5 .99

Safety LS Krogsrud

QR. Q. Ahucheleto for

814199

Environmental NA

Modification Projects

DS Tank Operations

Design Authority PF Kison
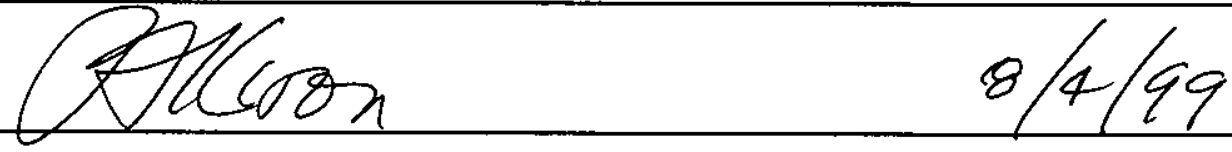

Design Engineer

Design Engineer

Design Engineer

Design Engineer 


\section{APPENDIX}

Planned ABU from Engineering Task Plan (HNF-3322)

\begin{tabular}{|c|c|c|c|c|c|}
\hline \multicolumn{6}{|c|}{ ACCEPTANCE FOR BENEFICIAL USE (ABU) } \\
\hline $\begin{array}{l}\text { 3. Document No. } \\
\text { HNF-3322 }\end{array}$ & $\begin{array}{l}\text { 4. Project No. } \\
\text { NA }\end{array}$ & $\begin{array}{l}\text { 5. Ssc } \\
\text { Designator } \\
\text { GS }\end{array}$ & $\begin{array}{l}\text { 6.System/BIdg/ } \\
\text { Facility } \\
241-S Y-101\end{array}$ & $\begin{array}{l}\text { 7.Equip/Component } \\
\text { No. }\end{array}$ & $\begin{array}{l}\text { 8. ECN/EDT No. } \\
\text { EDT } 140119\end{array}$ \\
\hline 9. Final ABU () & \multicolumn{2}{|c|}{ Partial ABU () } & \multicolumn{2}{|l|}{ 10. ATP Rerun Required } & ( ) No \\
\hline
\end{tabular}

11. Description of Work

Modify Neutron/Gamma Probes to operate with the existing LOW Surveillance Vans at Tank 241-SY-101 MITs in accordance with procedure TO-040-333.

This ABU documents the completion of all activities necessary for the deployment of modified neutron and gamma probes with the existing LOW vans at Tank 241-SY-101 MITs.

\begin{tabular}{|l|l|l|l} 
12. Description of Work to be Completed & $\begin{array}{l}\text { 13. Scheduled } \\
\text { Completion Date }\end{array}$ & $\begin{array}{l}\text { 14. Responsibility for } \\
\text { Completion }\end{array}$ & 15. Impact on Operations/Safety
\end{tabular}

bility for

\section{Funding 18. Document Number} Source
19. Responsibility for Completion
20. Required prior to final $A B U$ (Yes/No)

\section{TRAINING:}

\begin{tabular}{|c|c|c|c|c|}
\hline - Training manuals & () & & & \\
\hline Training to Operating Crews & $(x)$ & TBD & J. D. Adrian & Yes \\
\hline Training to Maintenance Crews & $(x)$ & TBD & J. D. Adrian & Yes \\
\hline
\end{tabular}

\section{ENGINEERING:}

\begin{tabular}{|lr|l|l|l|l|}
\hline - & $(\mathrm{x})$ & & HNF-3322 & N. S. Cannon & Yes \\
\hline - Engineering Task Plan & $($ ) & & & & \\
\hline - Final Safety Analysis Report (FSAR) & $($ ) & & & & \\
\hline - Safety Assessment (SA) & $(1)$ & & & & \\
\hline - Functional Design Criteria (FDC) & $(1)$ & & & & \\
\hline
\end{tabular}




\begin{tabular}{|c|c|c|c|c|}
\hline 16. Documents or Other Items/Tasks to be Updated & $\begin{array}{l}\text { 17. Funding } \\
\text { Source }\end{array}$ & 18. Document Number & $\begin{array}{l}\text { 19. Responsibility } \\
\text { for Completion }\end{array}$ & $\begin{array}{l}\text { 20. Required } \\
\text { prior to final } A B U \\
\text { (Yes/No) }\end{array}$ \\
\hline - Design Review Report ( ) & & & & \\
\hline - Conceptual Design Report (CDR) ( ) & & & & \\
\hline $\begin{array}{l}\text { - Supplemental Design Requirements Document } \\
\text { (SDRD) }\end{array}$ & & & & \\
\hline - $\quad$ System Design Description (SDD) & & & & \\
\hline $\begin{array}{l}\text { Test procedures/specifications (x) } \\
\text { Test Report }\end{array}$ & & $\begin{array}{l}\text { ATP/OTP \& OTR } \\
\text { (Document \# TBD) }\end{array}$ & N. S. Cannon & Yes \\
\hline - $\quad$ Safety Equipment List & & & & \\
\hline - Environmental Impact Statement & & & & \\
\hline - Environmental Report & & & & \\
\hline - Environmental Permits & & & & \\
\hline - $\quad$ Stress/Seismic Analysis & & & & \\
\hline - $\quad$ Stress/Design Report & & & & \\
\hline - Equipment Specification & & & & \\
\hline - Procurement Specification & & $\begin{array}{l}\text { Included in PR A31978 \& } \\
\text { A31980 }\end{array}$ & N. S. Cannon & Yes \\
\hline - Construction Specification & & & & \\
\hline - Essential Material Specification & & & & \\
\hline - Technical Specification & & & & \\
\hline - $\quad$ As-built Drawing & & TBD & P. R. Deichelborer & Yes \\
\hline - Interface Control Drawing & & & & \\
\hline - Computer Software & & & & \\
\hline \multicolumn{5}{|l|}{ OPERATIONS: } \\
\hline - Operating and Maintenance Manuals & & & & \\
\hline - $\quad$ Operating Procedures & & TO-040-333* & D. A. Barnes & Yes \\
\hline - Operation Instructions & & & & \\
\hline - Calibration Procedures & & TO-040-333* & D. A. Barnes & Yes \\
\hline - Preventative Maintenance Procedures & & & & \\
\hline - Operations Safety Requirements & & & & \\
\hline
\end{tabular}


HNF-4917, Rev. 0

\begin{tabular}{|c|c|c|c|c|}
\hline 16. Documents or Other Items/Tasks to be Updated & $\begin{array}{l}\text { 17. Funding } \\
\text { Source }\end{array}$ & 18. Docurnent Number & $\begin{array}{l}\text { 19. Responsibility } \\
\text { for Completion }\end{array}$ & $\begin{array}{l}\text { 20. Required } \\
\text { prior to final ABU } \\
\text { (Yes/No) }\end{array}$ \\
\hline \multicolumn{5}{|l|}{ QUALITY ASSURANCE: } \\
\hline - Inspection Plan & & TBD & R. R. True & Yes \\
\hline - $\quad$ QAPP & & & & \\
\hline - QAP JP & & & & \\
\hline - NEC Inspection & & & & \\
\hline \multicolumn{5}{|l|}{ PROCUREMENT ACTIVITIES: } \\
\hline - Vendor Information & & $\mathrm{TBD}$ & N. S. Cannon & Yes \\
\hline - $\quad$ Material List & & & & \\
\hline - $\quad$ Spare Parts & & & & \\
\hline - $\quad$ Purchase Requisition(s) & & PR A31978 \& A319830 & N. S. Cannon & Yes \\
\hline - $\quad$ ALARA Management Worksheet & & & & \\
\hline - Other Commercial Grade Item forms & & & & . \\
\hline
\end{tabular}

- Minor changes (if any) are expected for the procedures. 
HNF-4917, Rev. 0

PROJECTS: CHARACTERIZATION OPERATIONS AND ENGINEERING

Cog/Field Engineer DA Barnes

Cog/Field Engineer

Cog/Field Engineer

Cog/Field Mgr. NW Kirch

QA RR True

Safety LS Krogsrud

Environmental NA

Modification Projects

DS Tank Operations

Design Authority PF Kison

Design Engineer

Design Engineer

Design Engineer

Design Engineer

Design Engineer 
DISTRIBUTION SHEET

\begin{tabular}{|c|c|c|c|c|c|}
\hline \multirow{2}{*}{$\begin{array}{l}\text { To } \\
\text { Distribution }\end{array}$} & \multirow{2}{*}{\multicolumn{3}{|c|}{$\begin{array}{l}\text { From } \\
\text { Electrical I \& C }\end{array}$}} & \multirow{2}{*}{\multicolumn{2}{|c|}{$\begin{array}{l}\text { Page } 1 \text { of } 1 \\
\text { Date August } 3,1999\end{array}$}} \\
\hline & & & & & \\
\hline \multirow{2}{*}{\multicolumn{4}{|c|}{$\begin{array}{l}\text { Project Title/Work Order } \\
\text { Multifunction Instrument Tr } \\
\text { Acceptance for Beneficial }\end{array}$}} & \multirow{2}{*}{\multicolumn{2}{|c|}{$\begin{array}{ll}\text { EDT No. } & 140140 \\
\text { ECN No. } & \text { NA } \\
\end{array}$}} \\
\hline & & & & & \\
\hline Name & MSIN & $\begin{array}{l}\text { Text } \\
\text { With All } \\
\text { Attach. }\end{array}$ & Text Only & $\begin{array}{l}\text { Attach./ } \\
\text { Appendix } \\
\text { Only }\end{array}$ & $\begin{array}{c}\text { EDT/ECN } \\
\text { Only }\end{array}$ \\
\hline $\begin{array}{l}\text { J. D. Adrian } \\
\text { D. A. Barnes } \\
\text { R. E. Bauer } \\
\text { N. S. Cannon (3) } \\
\text { J. D. Criddle, Jr. } \\
\text { P. R. DeicheTbohrer } \\
\text { K. M. Hall } \\
\text { G. D. Johnson } \\
\text { N. W. Kirch } \\
\text { P. F. Kison } \\
\text { L. S. Krogsrud } \\
\text { D. C. Larsen } \\
\text { M. D. Rickenbach } \\
\text { D. Scott, Jr. } \\
\text { D. L. Sparks } \\
\text { F. S. Stong } \\
\text { R. R. True } \\
\text { R. P. Tucker } \\
\text { Central Files (1) + Orig. }\end{array}$ & $\begin{array}{l}\text { T4-01 } \\
\text { R2-12 } \\
\text { G1-54 } \\
\text { L6-38 } \\
\text { S7-12 } \\
\text { S7-12 } \\
\text { R2-12 } \\
\text { S7-73 } \\
\text { R2-11 } \\
\text { T4-07 } \\
\text { T4-07 } \\
\text { T4-08 } \\
\text { H3-28 } \\
\text { S5-07 } \\
\text { S5-03 } \\
\text { S7-12 } \\
\text { T4-07 } \\
\text { T4-07 } \\
\text { A3-88 }\end{array}$ & 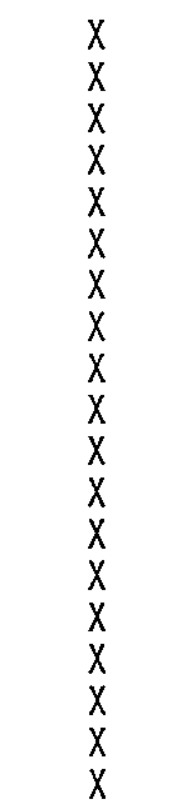 & & & \\
\hline
\end{tabular}

